\title{
No association between germline allele-specific expression of TGFBR I and colorectal cancer risk in Caucasian and Ashkenazi populations
}

\author{
N Seguí ${ }^{1,6}$, KN Stevens ${ }^{2,6}$, E Guinó ${ }^{3}$, LS Rozek ${ }^{4}$, VR Moreno ${ }^{3,5}$, G Capellá', SB Gruber,,2 and L Valle*,1 \\ 'Translational Research Laboratory, Catalan Institute of Oncology, IDIBELL, Hospitalet de Llobregat, Av. Gran Vía 199-203, Barcelona 08908, Spain; \\ ${ }^{2}$ Departments of Internal Medicine, Epidemiology, and Human Genetics, Division of Molecular Medicine and Genetics, University of Michigan, 1524 \\ BSRB, 109 Zina Pitcher, Ann Arbor, MI 48109, USA; ${ }^{3}$ Bioinformatics and Biostatistics Unit, Catalan Institute of Oncology, IDIBELL, Hospitalet de \\ Llobregat, Barcelona 08908, Spain; ${ }^{4}$ Environmental Health Sciences, School of Public Health, University of Michigan Medical School, Ann Arbor, MI \\ 48109, USA; ${ }^{5}$ Department of Clinical Sciences, Faculty of Medicine, University of Barcelona, Barcelona, Spain
}

BACKGROUND: Germline allele-specific expression (ASE) of the TGFBR I gene has been reported as a strong risk factor for colorectal cancer (CRC) with an odds ratio close to 9. Considering the potential implications of the finding, we undertook the task of validating the initial results in this study.

METHODS: Allele-specific expression was measured using the highly quantitative and robust technique of pyrosequencing. Individuals from two different populations were studied, one Caucasian-dominated and the other of Ashkenazi Jewish descent, with different sources of non-tumoral genetic material in each.

RESULTS: Our results showed no statistically significant differences in the degree of ASE between CRC patients and controls, considering ASE as either a quantitative or a binary trait. Using defined cutoff values to categorise ASE, $1.0 \%$ of blood lymphocytes from informative Israeli cases (total $n=96$ ) were ASE positive (median I.00; range 0.76-1.31) and 2.2\% of informative matched controls (total $n=90$ ) were ASE positive (median 1.00; range 0.76- 1.87). Likewise, normal mucosae from Spanish patients (median I.03; range: $0.68-1.43 ; n=75)$ did not show significant differences in the degree of ASE when compared with the Israeli patients or controls.

CONCLUSIONS: Taken together, these results suggest that ASE of TGFBR I does not confer an increased risk of CRC.

British Journal of Cancer (20II) I 04, 735-740. doi:I0.1038/sj.bjc.6606079 www.bjcancer.com

Published online II January 20 II

(C) 20II Cancer Research UK

Keywords: allele-specific expression; allelic imbalance; TGFBR /; colorectal cancer predisposition; pyrosequencing; cancer-predisposing gene

Colorectal cancer (CRC) is among the four most common cancers in industrialised countries, and is one of the leading causes of cancer-related deaths. Although familial clustering of CRC occurs in $20-30 \%$ of all cases, the known highly penetrant autosomaldominant and -recessive forms of the disease account for less than $5 \%$ of all CRC cases (de la Chapelle, 2004; Lynch et al, 2009). Although additional low-penetrance alleles have been proposed in the last few years, the underlying genetic risk factors for CRC predisposition remain largely unknown (Hemminki et al, 2009).

On the basis of previous evidence that pointed towards the importance of downstream signalling elements of the transforming growth factor $\beta$ (TGF- $\beta$ ) pathway in CRC (Wood et al, 2007), and the known linkage peak for familial CRC in 9q22-31 where TGFBR1 is located (Wiesner et al, 2003; Kemp et al, 2006;

*Correspondence: Dr L Valle; E-mail: Ivalle@iconcologia.net or Dr SB Gruber; E-mail: sgruber@med.umich.edu

${ }^{6}$ These authors contributed equally to this work.

Received 22 October 2010; revised 3 December 2010; accepted 6 December 2010; published online II January 201 I
Skoglund et al, 2006), we undertook the task of studying the role of TGFBR1 in CRC predisposition. Although risk-conferring germline genetic variants in this gene had not been identified, we reported that germline allele-specific expression (ASE) of TGFBR1, measured with the SNaPshot technique, occurred in $\sim 20 \%$ of informative CRC patients and $\sim 3 \%$ of informative controls, thus conferring a substantially increased risk of CRC (odds ratio 8.7, 95\% confidence interval (CI): 2.6-29.1) (Valle et al, 2008). This differential allele-specific expression was suggested to be dominantly inherited and to alter the downstream SMAD-mediated TGF- $\beta$ signalling (Valle et al, 2008). A subsequent report showed that $A P C^{\mathrm{Min} /+} ; \mathrm{Tgfbr}^{+/-}$mice developed twice as many intestinal tumours and colonic carcinomas as $A P C^{\mathrm{Min} /+} ; \mathrm{Tgfbr1}{ }^{+/+}$, supporting the role of TGFBR1 gene haploinsufficiency in CRC development (Zeng et al, 2009). Also, TGFBR1*6A, a common variant in exon 1 of the gene, has been weakly associated with CRC (Pasche et al, 2004; Skoglund et al, 2007).

As allele-specific expression of TGFBR1 has the potential to be used in the clinical evaluation of CRC risk, the aim of this study was to further investigate the extent of ASE of TGFBR1 in CRC using the robust and specific pyrosequencing technique for ASE 
determination. In addition, we studied two different populations with different biological sources of non-tumour genetic material to evaluate ASE frequency in a variety of populations.

\section{MATERIALS AND METHODS}

\section{Patients and controls}

Uncultured blood lymphocytes from a total of 426 Ashkenazi Jewish CRC patients and 433 Ashkenazi Jewish controls were obtained from a collection of Israeli CRC patients and matched controls. This series corresponds to a population-based casecontrol study (Molecular Epidemiology of Colorectal Cancer; MECC) of incident CRC, including histopathologically confirmed cases of all incident CRC diagnosed in northern Israel beginning 31 March 1998 (Poynter et al, 2005). Informed consent was obtained from all of the subjects who participated in the study. All 426 CRC patients showed tumour microsatellite stability and did not carry germline mutations in known cancer-predisposing genes.

A total of 178 normal mucosae from Spanish CRC patients (6\% showed tumour microsatellite instability) were obtained from a hospital-based case-control study (Bellvitge Colorectal Cancer Study; BCCS). Cases were consecutive patients with a new diagnosis of colorectal adenocarcinoma attending a University Hospital in Barcelona. Details about the study population, interviews and collection of biological samples were published elsewhere (Landi et al, 2003).

\section{Nucleic acid extraction and cDNA synthesis}

Genomic DNA from purified blood lymphocytes and frozen normal colon mucosa was extracted using standard phenolchloroform procedures. For total RNA extraction, the different tissue sources were processed with TRIzol reagent (Invitrogen, Carlsbad, CA, USA). In all cases, nucleic acid concentrations and purities were analyzed with the NanoDrop spectrophotometer, and the level of degradation of the RNA (RIN number) was checked by using the RNA Nano assay on the Agilent 2100 Bioanalyzer system (Agilent Technologies, Santa Clara, CA, USA) when necessary.

Total RNA was treated with DNAse (DNAfree, Ambion, Austin, TX, USA) before cDNA synthesis (Transcriptor First Strand cDNA Synthesis Kit, Roche Diagnostics GmbH, Mannheim, Germany).

\section{Genotyping of transcribed SNPs}

Current techniques for ASE determination require heterozygous markers in the transcribed regions of the gene to discriminate between its two alleles. The transcribed markers we used were four SNPs located in the $3^{\prime}$-UTR region: rs334349, rs420549, rs7850895 and rs1590. Owing to the fact that rs334349 and rs1590 are in strong linkage disequilibrium, only the latter was genotyped to determine the informativeness of both. Three commercially available TaqMan SNP genotyping assays were used to genotype rs420549 (C_662618_1), rs7850895 (C_29248567_20) and rs1590 (C_2945143_10) (Applied Biosystems Inc., Foster City, CA, USA). Reactions were performed following the instructions provided by the manufacturer.

\section{ASE determination by pyrosequencing}

PCR and pyrosequencing reactions for rs334349, rs1590 and rs420549 were performed as described previously (Guda et al, 2009). For rs7850895, PCR and sequencing primers were designed using the PSQ Assay Design software provided by the manufacturer: PCR-fw-5'-TCATGCCATATGTAGTTGCTGTAG-3'; biotinylated PCR-rv-5'-ACACCCCTAAGCATGTGGAGA-3' ${ }^{\prime}$; and SEQ- $5^{\prime}-\mathrm{C}$ CTAGTGCAAGTTACAATAT-3'. After PCR, DNA and cDNA amplification products were sequenced on a PyroMark MD pyrosequencing instrument (Qiagen, Chatsworth, CA, USA).

The proportions of individual alleles for each SNP were obtained from the PyroMark MD software calculations. The medians and standard deviations (s.d.) of the triplicates for both DNA and cDNA were calculated for each SNP. To obtain an ASE value, the ratio of the common $v s$ the rare allele in the cDNA was normalised to the respective ratio in the DNA: cDNA (median common allele/ median rare allele)/DNA (median common allele/median rare allele). The final ASE value was calculated as the median of the ASE values obtained for the SNPs studied in each sample.

Before the complete analysis of all informative samples, we tested the robustness and reproducibility of pyrosequencing compared with SNaPshot, the technique used in the original report (Valle et al, 2008), by randomly choosing 10 informative samples and measuring ASE using both techniques. SNaPshot was carried out as described previously (Valle et al, 2008) and ASE value calculations were performed as described above for both techniques. Pyrosequencing yielded lower variability in ASE among different SNP markers and it was able to obtain valuable results in situations when SNaPshot was not able to assess ASE (Supplementary Table 1). Guda et al (2009) previously reported additional information showing that ASE results obtained by pyrosequencing can be reproduced by SNaPshot, supporting the ability of pyrosequencing to detect allelic imbalances.

\section{Statistical analyses}

Pairwise comparisons between cases and controls were performed using the Wilcoxon's rank-sum test and Bonferroni correction was applied to account for the two comparisons performed: MECC cases vs MECC controls and MECC cases vs BCCS cases.

To dichotomise the ASE variable, cutoff points were established based on the ASE values obtained in cancer-free controls (median $\pm(2 \times$ s.d. $))$. When ASE was considered as a binary variable, comparisons of proportions between cases and controls were performed using a likelihood ratio test derived from logistic regression adjusting for population source.

\section{RESULTS}

Among a total of 426 Ashkenazi Jewish CRC patients from the Israeli MECC study, $115(27 \%)$ were informative for at least one SNP tested: $88(20.7 \%)$ for $r 3334349$ and rs1590, 65 (15.3\%) for rs420549 and $18(4.2 \%)$ for rs7850895. Of a total of 433 Ashkenazi Jewish MECC controls, $112(25.9 \%)$ were informative: $81(18.7 \%)$ for rs334349 and rs1590, 59 (13.6\%) for rs420549 and 19 (4.4\%) for rs7850895. Of 178 normal mucosae from a Spanish collection of Caucasian CRC patients (BCCS), $88(49.4 \%)$ were heterozygous for at least one SNP genotyped: $64(36 \%)$ for rs334349 and rs1590, 64 (36\%) for rs420549 and $18(10 \%)$ for rs7850895. Three of the 88 had no RNA available.

ASE values were obtained for 96 (83.5\%) informative MECC CRC patients, $90(80.4 \%)$ informative MECC controls and 75 (85.2\%) informative BCCS CRC patients. The ASE values obtained for cases and controls are shown in Figure 1. For the MECC series alone, values range from 0.76 to 1.31 (median: 1.00 ) in cases, and from 0.76 to 1.87 (median: 1.00) in controls (Figure 1A). When ASE was considered as a continuous variable, no differences were detected between cases and controls (median difference -0.002 ; $95 \% \mathrm{CI}:-0.027$ to $0.032 ; P=0.86$ ). Although observed data suggest that ASE is a quantitative trait, ASE was transformed into a binary trait (ASE $v s$ non-ASE) to facilitate the interpretation of the results. For this purpose, cutoff points were defined based on the results obtained in controls (median $\pm(2 \times$ s.d $)=1.00 \pm(2 \times 0.157))$. Under that criterion, $1.0 \%$ ( 1 out of 96) of informative CRC patients and $2.2 \%$ ( 2 out of 90) of informative controls showed ASE of TGFBR1 $(P=0.52)$ (Figure 2). 

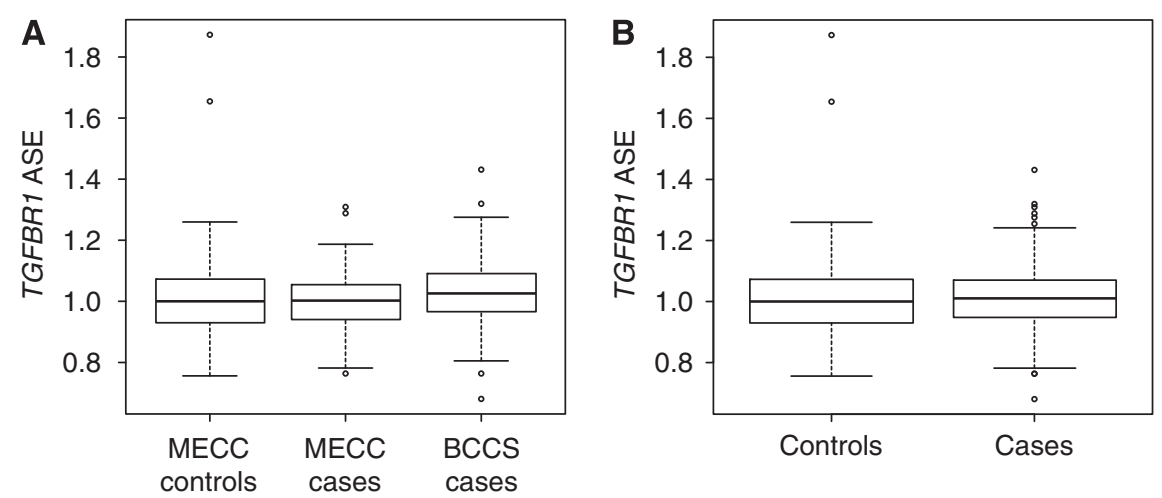

Figure I TGFBR I ASE distributions in cases and controls. (A) TGFBR I ASE in MECC controls $(n=90)$, MECC CRC patients $(n=96)$ and BCCS CRC patients $(n=75)$. (B) TGFBRI ASE in CRC patients $(n=17 \mathrm{I})$ and controls $(n=90)$. The boxes represent the inter-quartile range of distributions $(25-75$ th percentile); the horizontal lines within the boxes represent the medians; and the vertical lines represent the 5 and 95 th percentiles.

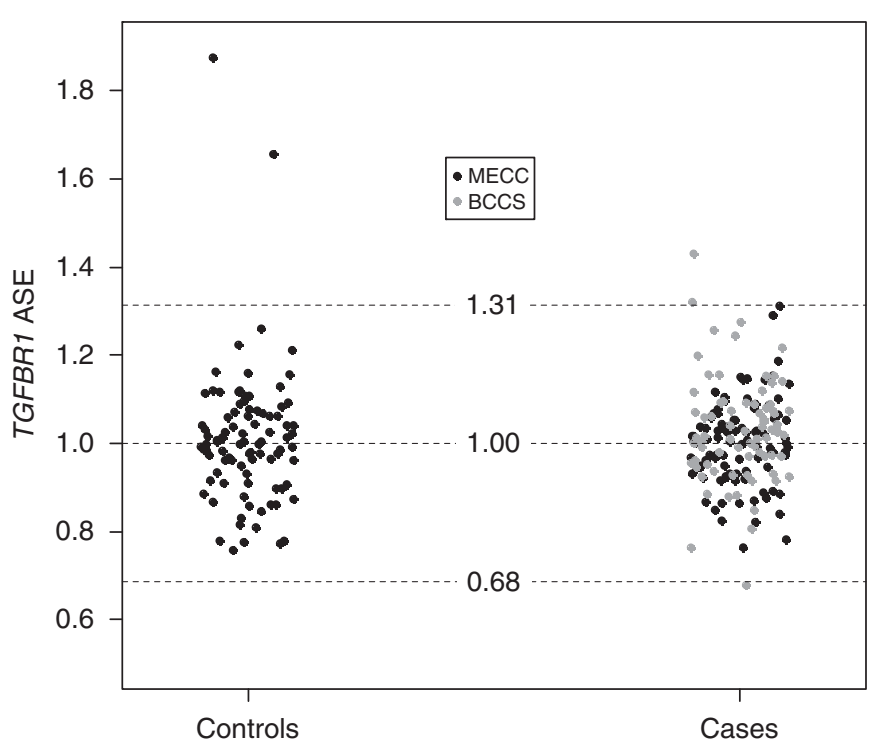

Figure 2 TGFBRI ASE distribution in I7I CRC patients (96 MECC (black dots) and 75 BCCS (grey dots) CRC patients) and 90 controls. The median and cutoff points, defined as the median $\pm 2 \times$ s.d. of controls, used to categorise ASE are indicated as discontinuous lines.

No differences in ASE levels were identified between MECC CRC patients (median 1.00; range 0.76-1.31) and BCCS patients (median 1.03; range $0.68-1.43$ ) (median difference 0.026 ; $95 \%$ CI: -0.001 to $0.059 ; P=0.06$ ). Consistent with this analysis, no differences were identified between MECC and BCCS CRC patients when ASE was treated as a binary variable $(P=0.20)$, suggesting that ethnic origin (Ashkenazi Jewish and Caucasian) and the source of biological material assessed (uncultured blood lymphocytes and normal colon mucosae) do not have a major influence on TGFBR1 ASE assessment. This allowed us to combine the two groups of CRC patients and compare them with the available group of controls (Figures 1B and 2). Combining all data from MECC and BCCS subjects, no differences were detected between CRC patients and controls when considering ASE as either a quantitative (median difference -0.010 ; 95\% CI: -0.037 to $0.017 ; P=0.48$ ) or a binary variable ( $P=0.52$, adjusted by population).

The RNA quality of subjects with ASE in both the BCCS and MECC series was checked to confirm that they had been classified as such owing to the presence of real allelic imbalances and not owing to technical artefacts caused by poor RNA quality. In all samples where the source RNA was available, the RIN value was above 6. To ensure that our results were not affected by poor performance of the PCR/pyrosequencing reaction owing to low RNA quality, a more stringent analysis was carried out, including only those samples whose s.d. among pyrosequencing triplicates was below 0.20 . Similar to the results using the entire sample, no differences were detected between CRC patients and controls (Supplementary Figure 1).

\section{DISCUSSION}

The presence of allelic imbalances is well known to be widespread throughout the transcriptome and has been associated with cancer risk in some instances (Yan et al, 2002; Raval et al, 2007; Chen et al, 2008). In a previous report, we suggested that ASE of TGFBR1 confers a substantially increased risk of CRC (odds ratio 8.7), potentially placing ASE of TGFBR1 among the major contributors to the genetic predisposition to both familial and sporadic CRC (Valle et al, 2008). The main significance of those findings pertains to early detection and prevention of CRC, therefore requiring validation in larger series and different populations for future implementation in clinical practice. Here, using a more robust technique for ASE determination, studying Ashkenazi Jewish and Caucasian populations, and using different sources of non-tumoral genetic material, we identified no differences in the degree or frequency of ASE of TGFBR1 between CRC patients and controls, discarding its role in CRC predisposition. Our results are supported by the study by Guda et al (2009), in which it was concluded that ASE of TGFBR1 is unlikely to be the major driver of linkage in some colon neoplasia families to the $9 q 22.2-31.2$ region, in which TGFBR1 is located, and that ASE is not associated with sporadic CRC $(n=44)$. Recently, Carvajal-Carmona et al (2010) reported no evidence of genetic variation at TGFBR1 as a predisposing factor for CRC and found no increased level of TGFBR1 ASE in 24 familial CRC patients compared with 45 informative controls. In fact, ASE turned out to be more prevalent among controls than among cases.

Very recently, two additional studies on ASE of TGFBR1 were published. In the first study, ASE of TGFBR1, assessed by SNaPshot, was found in approximately $10 \%$ of CRC patients (15\% of informative patients), agreeing with our initial report; however, no controls were included for comparison (Pasche et al, 2010). In the second study, where ASE was measured by pyrosequencing, 109 informative cases and 125 informative controls were studied. No differences were identified when ASE was considered as a binary variable; however, when treated with a continuous variable, ASE was significantly higher in cases than in controls. However, the differences identified between CRC patients and controls were very subtle and definitely not useful for cancer risk assessment (Tomsic et al, 2010). Table 1 shows the main 
Table I Summary of the characteristics and results obtained in different studies on ASE of TGFBRI and CRC risk

\begin{tabular}{|c|c|c|c|c|c|c|c|}
\hline \multirow[b]{2}{*}{ Study } & \multirow[b]{2}{*}{ Population } & \multirow[b]{2}{*}{$\begin{array}{l}\text { Nucleic acid } \\
\text { source }\end{array}$} & \multirow[b]{2}{*}{ Methodology } & \multirow[b]{2}{*}{$\begin{array}{l}\text { Allelic } \\
\text { markers }\end{array}$} & \multirow[b]{2}{*}{$\begin{array}{l}\text { Informative } \\
\text { cases; controls }\end{array}$} & \multicolumn{2}{|c|}{ ASE: Cases vs controls ${ }^{a}$} \\
\hline & & & & & & Quantitative & $\begin{array}{l}\text { Binary } \\
\text { (ASE vs non-ASE) }\end{array}$ \\
\hline Valle et al (2008) & $\begin{array}{l}C \sim 90 \% \\
O \sim 10 \%\end{array}$ & Peripheral blood & SNaPshot & $\begin{array}{l}\text { rs334348 } \\
\text { rs } 787 \mid 490 \\
\text { rs334349 } \\
\text { rs } 1590\end{array}$ & 138; 105 & $\begin{array}{l}P=0.12 \text { Wilcoxon } \\
\text { test } \\
P=0.02 \\
\text { Permutation test }\end{array}$ & $\begin{array}{l}\text { Cutoff: }<0.67, \geqslant 1.5 \\
\text { (ROC analysis) } \\
\text { ASE: } 29 / 138 ; 3 / 105 \\
P=7.66 \times 10^{-5}\end{array}$ \\
\hline $\begin{array}{l}\text { Carvajal- } \\
\text { Carmona et al } \\
(2010)\end{array}$ & $C \sim 100 \%$ & $\begin{array}{l}\text { Lymphoblastoid } \\
\text { cell line (EBV) }\end{array}$ & $\begin{array}{l}\text { Genescan } \\
\text { SNaPshot }\end{array}$ & $\begin{array}{l}* 6 \mathrm{~A} / 9 \mathrm{~A} \\
\text { rs } 1590\end{array}$ & Familial: 24; 45 & $\begin{array}{l}P=0.09 \text { Wilcoxon } \\
\text { test }^{\mathrm{b}} \\
P=0.13 \text { t-test }^{\mathrm{b}}\end{array}$ & $\begin{array}{l}\text { Cutoff: }<0.67, \geqslant 1.5 \\
\text { (Valle et al, 2008) } \\
\text { ASE: } 7 / 24 ; 12 / 45 \\
P=0.83\end{array}$ \\
\hline $\begin{array}{l}\text { Tomsic et al } \\
(2010)\end{array}$ & $\begin{array}{l}\text { C 92\% } \\
\text { AA } \sim 8 \%\end{array}$ & Peripheral blood & Pyrosequencing & $\begin{array}{l}\text { rs868 } \\
\text { rs334348 } \\
\text { rs334349 } \\
\text { rs420549 } \\
\text { rs } 1590\end{array}$ & $109 ; 125^{c}$ & $\begin{array}{l}P=0.009 ; 0.006 \\
\text { Wilcoxon test } \\
P=0.081 ; 0.077 \\
\text { Permutation test } \\
P=0.003 ; 0.007 \\
\text { Permutation } \\
\text { (median) }\end{array}$ & $\begin{array}{l}\text { Cutoff: }<0.67, \geqslant 1.5 \\
\text { (Valle et al, 2008) } \\
\text { ASE: } 2 / 109 ; 2 / 125 \\
\text { Cutoff: }<0.9, \geqslant 1.1 \\
\text { (ROC analysis) } \\
\text { ASE: } 51 / 109 ; 39 / 125 \\
P=0.06\end{array}$ \\
\hline This study & $\begin{array}{l}\text { Ash } 100 \% \\
\text { C } 100 \%\end{array}$ & $\begin{array}{l}\text { Uncultured } \\
\text { lymphocytes } \\
\text { (Ash) } \\
\text { Normal colon } \\
\text { mucosa (C) }\end{array}$ & Pyrosequencing & $\begin{array}{l}\text { rs334349 } \\
\text { rs7850895 } \\
\text { rs420549 } \\
\text { rs } 1590\end{array}$ & $\begin{array}{l}\text { Ash: } 96 ; 90 \\
\text { C: } 75 ; 0\end{array}$ & $\begin{array}{l}\text { Ash: } P=0.86 \\
\text { Wilcoxon (median) } \\
\text { Ash+C: } P=0.48 \\
\text { Wilcoxon (median) }\end{array}$ & $\begin{array}{l}\text { Cutoff: }<0.67, \geqslant 1.5 \\
\text { (Valle et al, 2008) } \\
\text { Ash ASE: 0/96; 2/90 } \\
\text { Ash+C ASE: 0/171; } 2 / 90 \\
\text { Cutoff: <0.68, } \geqslant 1.31 \\
\text { (median } \pm 2 \times \text { s.d.) } \\
\text { Ash ASE: } 1 / 96 ; 2 / 90 \\
P=0.52 \\
\text { Ash+C ASE: 4/I7I; 2/90 } \\
P=0.52\end{array}$ \\
\hline
\end{tabular}

Abbreviations: $A A=$ African-American; $A S E=$ allele-specific expression; $A$ sh $=$ Ashkenazi lewish; $C=C$ aucasian; $C R C=$ colorectal cancer; $E B V=E p s t e i n-B a r r$ virus; $N A=$ not

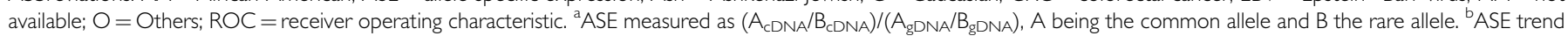
was more pronounced in the controls than in the cases. 'Forty-nine cases were the same as in Valle et al (2008).

characteristics and results of previous studies focused on the role of ASE of TGFBR1 in CRC risk.

Several features differentiate the original and present studies, and important consequences might have derived from these differences. The use of different assays for ASE determination and the exclusion of cases that showed high variability among replicates (exclusion of samples with low-quality source RNA shown in Supplementary Material) have likely increased the robustness of our study. Tomsic et al (2010) also found that the $\mathrm{SNaPshot}$ technology used for ASE determination gave inconsistent results, as evidenced by considerably larger standard deviations compared with pyrosequencing, and that high RNA quality is essential for reproducibility of ASE.

Some SNP markers and sources of nucleic acids used were also different. The rs7871490 SNP, located in the $3^{\prime}$-UTR of TGFBR1, was used as a marker in the original study (Valle et al, 2008), but not in the present one. We previously found that the marker was very useful because it allowed us to significantly increase the number of informative individuals from 40 to $55-60 \%$. This SNP is located in a region of repetitive sequence, $5^{\prime}$-GGGGGTTTTTTTT TTGTTTTTTTTTT[G/T]TTGTTGTTGTTTTTGGGCCATTTCT-3', which might have affected the correct performance of SNaPshot owing to the design and molecular basis of the technique. When excluding all individuals from the original study whose ASE value was only based on the results obtained from the analysis of rs7871490 ( 16 out of 29 CRC patients and 3 out of 3 controls with ASE values >1.5), the proportion of ASE in informative CRC patients drops from $21 \%$ (29 out of 138 ) to $13 \%$ (13 out of 97 ) and in informative controls from $3 \%$ ( 3 out of 105) to $0 \%$ ( 0 out of 76 ). Likely because of the repetitive sequences in the flanking region of rs7871490, we were not able to design a pyrosequencing assay, which precluded a direct comparison between SNaPshot and pyrosequencing. A subset of individuals within this group belonged to the so-called 'group 2', which was characterised by a particular haplotype significantly over-represented among ASE CRC patients (Valle et al, 2008). To increase the number of informative individuals, the rs420549 and rs7850895 allelic markers were included in this study. This resulted in an increase of 22 out of $75(29 \%)$ informative BCCS CRC patients (three of which showed ASE), 22 out of 96 (23\%) informative MECC CRC patients (one showed ASE) and 28 out of 90 (31\%) informative MECC controls (two showed ASE). In short, all ASE individuals were informative for only either rs420549 or rs7850895. This observation, together with what has been discussed above about rs7871490, suggests that ASE might be more common among individuals who carry minor alleles for specific TGFBR1 SNPs, and 
therefore might be more or less frequent depending on the panel of SNP markers used to define informative individuals. Nevertheless, our results point to a similar frequency of ASE among cases and controls.

The possibility of ASE being tissue specific has been suggested previously (Cowles et al, 2002; Wilkins et al, 2007). This was one concern that arose in the paper from Guda et al (2009), in which the sources of nucleic acids for ASE determination were EBV-transformed cultured lymphocytes and normal mucosae from CRC patients, in contrast to the total blood used in our initial study (Valle et al, 2008). Carvajal-Carmona et al (2010) also employed lymphoblastoid cell lines. It is still unknown whether EBV transformation and/or cell culture alter the allelic expression of genes. Here, we obtained uncultured blood lymphocytes from the MECC series, which may well correlate with the total blood used in our previous study (Valle et al, 2008) or by Tomsic et al (2010), and normal mucosae from BCCS patients, which can be compared with the sporadic cases reported in the series from Guda et al (2009). These results suggest that different (uncultured) biological sources of genetic material for the determination of TGFBR1 ASE can be used without distinction.

In the original report, a mostly Caucasian population from Central Ohio was evaluated, whereas Ashkenazi Israeli and Caucasian Spanish populations were studied here. The fact that ASE first seemed to be heavily dependent on allele frequencies left open the possibility of inter-ethnic variation. The degree of SNP informativity was different between the two populations $(20 \%$ MECC vs 36\% BCCS for rs 334349 and rs $1590 ; 14 \%$ MECC vs $36 \%$ BCCS for rs420549; and 4\% MECC vs 10\% BCCS for rs7850895); however, no differences were detected in the level of ASE between the two populations. Unfortunately, we did not have access to all types of samples (normal mucosae and lymphocytes) from the same individuals or populations; therefore, there remains a certain degree of uncertainty about tissue and ethnic variability.
In conclusion, the improved determination of ASE of TGFBR1 achieved by pyrosequencing revealed no differences between CRC cases and controls, in both Caucasian and Ashkenazi populations. The sample size in ASE studies is highly relevant owing to their dependence on marker informativity and to the difficulties associated with collection of high-quality germline RNA. Finally, the use of different sources of non-tumour nucleic acids for ASE determination adds consistency to our results. However, the lack of informativity for transcribed SNPs in a substantial proportion of individuals complicates the task to assess the extent of germline ASE of TGFBR1 in CRC. New technological advances that allow the measurement of allelic imbalances in a more precise and informative manner will be of substantial importance to provide a definitive answer to the real extent of ASE at TGFBR1 in CRC patients.

\section{ACKNOWLEDGEMENTS}

We thank Dr Albert de la Chapelle for helpful discussions and Gemma Aiza for technical support. This work was partly funded by the Spanish Ministry of Science and Innovation (BFU200910281 to LV and SAF2009-07319 to GC); the Scientific Foundation of Asociación Española Contra el Cáncer; the National Institutes of Health (R01 CA81488-04 to SBG); the University of Michigan's Cancer Center (5 P30 CA46592 to SBG); Spanish Networks RTICCC (RD06/0020/1050); Gastroenterology Foundation Dr Francisco Vilardell (F05-01); and Carlos III Health Institute (through Acción Transversal del Cáncer, CIBERESP and Grants FIS PI08/1635, FIS PI08/1359, FIS 06/0545 and FIS 05/1006). NS is supported by a fellowship and LV holds a contract 'Ramón y Cajal', both from the Spanish Ministry of Science and Innovation.

Supplementary Information accompanies the paper on British Journal of Cancer website (http://www.nature.com/bjc)

\section{REFERENCES}

Carvajal-Carmona LG, Churchman M, Bonilla C, Walther A, Lefevre JH, Kerr D, Dunlop M, Houlston R, Bodmer WF, Tomlinson I. Comprehensive assessment of variation at the transforming growth factor beta type 1 receptor locus and colorectal cancer predisposition. Proc Natl Acad Sci USA (2010); 107: $7858-7862$

Chen X, Weaver J, Bove BA, Vanderveer LA, Weil SC, Miron A, Daly MB, Godwin AK (2008) Allelic imbalance in BRCA1 and BRCA2 gene expression is associated with an increased breast cancer risk. Hum Mol Genet 17: $1336-1348$

Cowles CR, Hirschhorn JN, Altshuler D, Lander ES (2002) Detection of regulatory variation in mouse genes. Nat Genet 32: $432-437$

de la Chapelle A (2004) Genetic predisposition to colorectal cancer. Nat Rev Cancer 4: 769-780

Guda K, Natale L, Lutterbaugh J, Wiesner GL, Lewis S, Tanner SM, Tomsic J, Valle L, de la Chapelle A, Elston RC, Willis J, Markowitz SD (2009) Infrequent detection of germline allele-specific expression of TGFBR1 in lymphoblasts and tissues of colon cancer patients. Cancer Res 69: $4959-4961$

Hemminki K, Forsti A, Lorenzo Bermejo J (2009) Surveying the genomic landscape of colorectal cancer. Am J Gastroenterol 104: 789-790

Kemp ZE, Carvajal-Carmona LG, Barclay E, Gorman M, Martin L, Wood W, Rowan A, Donohue C, Spain S, Jaeger E, Evans DG, Maher ER, Bishop T, Thomas H, Houlston R, Tomlinson I (2006) Evidence of linkage to chromosome 9 q22.33 in colorectal cancer kindreds from the United Kingdom. Cancer Res 66: 5003-5006

Landi S, Moreno V, Gioia-Patricola L, Guino E, Navarro M, de Oca J, Capella G, Canzian F (2003) Association of common polymorphisms in inflammatory genes interleukin (IL)6, IL8, tumor necrosis factor alpha, NFKB1, and peroxisome proliferator-activated receptor gamma with colorectal cancer. Cancer Res 63: 3560-3566
Lynch HT, Lynch PM, Lanspa SJ, Snyder CL, Lynch JF, Boland CR (2009) Review of the Lynch syndrome: history, molecular genetics, screening, differential diagnosis, and medicolegal ramifications. Clin Genet 76: $1-18$

Pasche B, Kaklamani V, Hou N, Young T, Rademaker A, Peterlongo P, Ellis N, Offit K, Caldes T, Reiss M, Zheng T (2004) TGFBR $1 * 6 A$ and cancer: a meta-analysis of 12 case-control studies. J Clin Oncol 22: $756-758$

Pasche B, Wisinski KB, Sadim M, Kaklamani V, Pennison MJ, Zeng Q, Bellam N, Zimmerman J, Yi N, Zhang K, Baron J, Stram DO, Hayes MG (2010) Constitutively decreased TGFBR1 allelic expression is a common finding in colorectal cancer and is associated with three TGFBR1 SNPs. J Exp Clin Cancer Res 29: 57

Poynter JN, Gruber SB, Higgins PD, Almog R, Bonner JD, Rennert HS, Low M, Greenson JK, Rennert G (2005) Statins and the risk of colorectal cancer. N Engl J Med 352: 2184-2192

Raval A, Tanner SM, Byrd JC, Angerman EB, Perko JD, Chen SS, Hackanson B, Grever MR, Lucas DM, Matkovic JJ, Lin TS, Kipps TJ, Murray F, Weisenburger D, Sanger W, Lynch J, Watson P, Jansen M, Yoshinaga Y, Rosenquist R, de Jong PJ, Coggill P, Beck S, Lynch H, de la Chapelle A, Plass C (2007) Downregulation of death-associated protein kinase 1 (DAPK1) in chronic lymphocytic leukemia. Cell 129: $879-890$

Skoglund J, Djureinovic T, Zhou XL, Vandrovcova J, Renkonen E, Iselius L, Bisgaard ML, Peltomaki P, Lindblom A (2006) Linkage analysis in a large Swedish family supports the presence of a susceptibility locus for adenoma and colorectal cancer on chromosome 9q22.32-31.1. J Med Genet 43: e7

Skoglund J, Song B, Dalen J, Dedorson S, Edler D, Hjern F, Holm J, Lenander C, Lindforss U, Lundqvist N, Olivecrona H, Olsson L, Pahlman L, Rutegard J, Smedh K, Tornqvist A, Houlston RS, 
Lindblom A (2007) Lack of an association between the TGFBR $1 * 6 \mathrm{~A}$ variant and colorectal cancer risk. Clin Cancer Res 13: 3748-3752

Tomsic J, Guda K, Liyanarachchi S, Hampel H, Natale L, Markowitz SD, Tanner SM, de la Chapelle A. Allele-specific expression of TGFBR1 in colon cancer patients. Carcinogenesis (2010); 31(10): $1800-1804$

Valle L, Serena-Acedo T, Liyanarachchi S, Hampel H, Comeras I, Li Z, Zeng Q, Zhang HT, Pennison MJ, Sadim M, Pasche B, Tanner SM, de la Chapelle A (2008) Germline allele-specific expression of TGFBR1 confers an increased risk of colorectal cancer. Science 321: 1361-1365

Wiesner GL, Daley D, Lewis S, Ticknor C, Platzer P, Lutterbaugh J, MacMillen M, Baliner B, Willis J, Elston RC, Markowitz SD (2003) A subset of familial colorectal neoplasia kindreds linked to chromosome 9q22.2 -31.2. Proc Natl Acad Sci USA 100: 12961-12965

Wilkins JM, Southam L, Price AJ, Mustafa Z, Carr A, Loughlin J (2007) Extreme context specificity in differential allelic expression. Hum $\mathrm{Mol}$ Genet 16: $537-546$
Wood LD, Parsons DW, Jones S, Lin J, Sjoblom T, Leary RJ, Shen D, Boca SM, Barber T, Ptak J, Silliman N, Szabo S, Dezso Z, Ustyanksky V, Nikolskaya T, Nikolsky Y, Karchin R, Wilson PA, Kaminker JS, Zhang Z, Croshaw R, Willis J, Dawson D, Shipitsin M, Willson JK, Sukumar S, Polyak K, Park BH, Pethiyagoda CL, Pant PV, Ballinger DG, Sparks AB, Hartigan J, Smith DR, Suh E, Papadopoulos N, Buckhaults P, Markowitz SD, Parmigiani G, Kinzler KW, Velculescu VE, Vogelstein B (2007) The genomic landscapes of human breast and colorectal cancers. Science 318: $1108-1113$

Yan H, Dobbie Z, Gruber SB, Markowitz S, Romans K, Giardiello FM Kinzler KW, Vogelstein B (2002) Small changes in expression affect predisposition to tumorigenesis. Nat Genet 30: 25-26

Zeng Q, Phukan S, Xu Y, Sadim M, Rosman DS, Pennison M, Liao J, Yang GY, Huang CC, Valle L, Di Cristofano A, de la Chapelle A, Pasche B (2009) Tgfbr1 haploinsufficiency is a potent modifier of colorectal cancer development. Cancer Res 69: 678-686 